\title{
The forensic psychotherapist: dying breed or evolving species?
}

\author{
Gwen Adshead, Senior Registrar, Department of Mental Health Sciences, \\ St George's Hospital Medical School, London SW17 0RE
}

My paper deals with the provision of psychotherapy services for forensic patients and the training available to the would-be forensic psychotherapist. Psychotherapy in the context of this paper refers to psycho-analytically based psychotherapy, either individual or group, and not to behavioural or cognitive models. In addition, two aspects of psychotherapy should be borne in mind: supportive psychotherapy in the context of ongoing therapeutic relationships (e.g. patients suffering from chronic mental illness) and mutative psychotherapy, which aims to change some aspect of the person. Both aspects are important in the practice of forensic psychiatry, perhaps especially the latter.

It will be suggested that both training opportunities and service provision reveal deep polarities in attitude, both conscious and unconscious, and that, just as many forensic clients do, 'splitting' mechanisms have been used to maintain the internal equilibrium. One of the results of this has been that the practice of psychotherapy with forensic clients is being done by consultant psychotherapists and other disciplines with an interest in forensic work, rather than forensic psychiatrists. I will examine current trends.

It may be helpful to look at the structure of training for forensic psychiatrists and their experience of psychotherapy. The forensic psychiatric trainee spends four years in training, and must have obtained MRCPsych before starting. At the beginning of training the forensic trainee will have had exposure to psychodynamic theory and psychotherapeutic practice, but the degree of exposure varies from place to place and individual to individual. Some trainees may have treated individuals or run groups under supervision for two or three years; some trainees may have had no practical psychotherapeutic experience.

Thus when trainees enter forensic training schemes their experience and aptitude will differ. The Royal College Training Committee guidelines regarding the training of forensic psychiatrists state that:

"the trainee should have acquired competence in the psychotherapeutic treatment of behavioural disorders"

(Psychotherapy in this case refers to all types of psychotherapy, not just psycho-analytically based.)

For example, the guidelines suggest that:

"Counselling with individuals or supportive groups, as well as simple explorative or cognitive psychotherapy, without attempting psychodynamic interpretation, will be sufficient for most trainees."

It is not clear exactly what is being referred to here. Clearly there are different levels of psychodynamic interpretation, depending on the type of therapeutic relationship, but I suggest that it is implausible to think that no interpretation is required by the therapist, since without interpretation it is hard to understand what is happening to the patient. I suggest that interpretation goes on all the time, even if it is not acknowledged as such. Further, one must question the supposition that counselling/supportive work requires less training and supervision. This view of psychotherapy can only make a hard task harder, and not surprisingly this can lead to forensic trainees focusing on other types of forensic work, while the psychotherapy is done by other professionals. This, in turn, reinforces the 'split' between psychotherapy and mainstream forensic psychiatry.

A survey of the 35 current forensic trainees examined their experience and attitudes to psychoanalytical psychotherapy (bearing in mind that for most of them this does not entail personal therapy or specific training, but rather an approach to treatment). These trainees are working in a variety of forensic settings, with the 14 health regions of England and Wales. Since the Butler Committee report of 1975 most regions have a medium secure unit, either in the form of one centralised unit or two or three smaller units. These units provide in-patient and outpatient services as well as community care, rehabilitation and consultation services. Trainees are also expected to work in prisons and maximum security (so-called 'special') hospitals, as well as doing medico-legal work. There is a shortage of forensic consultants, so that trainees are often under pressure in order to maintain a service.

The questionnaire examined the experience of different types of psychotherapy to which the trainees had been exposed. It also asked about teaching received, and their views in relation to possible courses. Responses were received from $67 \%$ of the trainees.

Eighty per cent (20) of responders had experience of using psychotherapy, in the general sense of psychological treatment. Of these, 16 had individual clients and five had run groups. The structure and focus of this work depended very much on the 
supervision available. Only two of 25 responders did not wish to do this type of work, and one said he thought psychotherapy was irrelevant to forensic work. Only two had requested experience in psychotherapy and not had it provided. Seventy-two per cent had some formal teaching, and the remainder said they would like some. In relation to psychotherapy, $84 \%$ said they thought "psychotherapy" should be part of forensic training, but only $20 \%$ thought personal therapy should be included. Twenty-eight per cent were undecided, feeling that it should be a personal decision for the trainee, and $52 \%$ definitely thought personal therapy should not be included.

In a general sense these results are very heartening, particularly in relation to the desirability of psychotherapeutic experience within forensic training. Nevertheless, that so many should reject personal therapy suggests again that formal psychoanalytic psychotherapy is seen as something completely separate from forensic practice. These figures suggest an enthusiasm at training level which is not manifested in research activities or service provision.

The questionnaire also specifically asked about the trainees' knowledge of the Portman Clinic. The Portman is a centre of excellence, providing psychoanalytic psychotherapy services for violent or sexual offenders. They also provide a valuable teaching resource in the form of seminars and lectures, and since October 1990 has been the base for a Diploma Course in Forensic Psychotherapy, which is the first of its kind.

Fourteen of the responders had heard of the course. Only $20 \%$ said they were interested in attending the course, while $68 \%$ said they were not interested. The following reasons were given for this lack of interest.

(a) Too far away. Clearly, since some of the respondents worked in places geographically distant from the Portman, this was not unexpected.

(b) Not relevant. This only applied to two people, one saying that the validity of the Portman's methods had not been established(!).

(c) Lack of time. Nearly a third of "not interested" respondents said that, although the course in principle interested them, they felt the claims on their time by other training requirements made this impossible.

These views are borne out when the applications for the Portman course are broken down into professional groups. Only two out of 20 applications came from senior registrars in forensic psychiatry. What this suggests is that, while forensic psychotherapy is seen as relevant and desirable as part of training, other aspects of training are seen as having priority. (It should be said that the Portman course requires the attendants to be in personal therapy.)
The split between psychotherapy and forensic psychiatry further manifests itself in the provision of psychotherapeutic services for forensic clients. For example, not every medium secure unit has access to a psychotherapist. This lack may lead to the unit's refusal to admit and treat personality disordered patients. This can then become part of a folk-lore that personality disordered patients are not treatable, when the truth is that the unit lacks the appropriate staff. Thus the patients become 'offenders' once again; it is they who have the untreatable condition, not the carers, who are in a 'non-treating' mode. This is also seen in the out-patient clinic, where psychotherapy is seen as too demanding in terms of time for too little obvious gain. Leaving aside the vexed issue of what it means to be 'better', in both forensic and psychotherapeutic terms, such a view can lead to patients being rejected and abused by the service that was designed to help them; a direct repeat of their previous experiences.

A further example relates to prison work. There are visiting psychotherapists, but very few; the bulk of the work is done by visiting psychiatrists, who see and assess those prisoners referred by the prison staff, or those who ask to see a psychiatrist. Most visiting psychiatrists have only a session of two or three hours a week; little time to think about psychotherapy, let alone provide it. The prison medical staff, both doctors and warders, seem to feel very ambivalent about the provision of such treatment for prisoners; on the one hand they are the designated carers, and recognise their patients' suffering; on the other, the psychiatrist's time is seen as something very good, that 'bad' prisoners should not receive. This, in part, reflects their need (and ours, perhaps) to maintain the split between themselves as 'good' custodians and the men as 'bad' prisoners. However, it also encourages the provision of psychotherapeutic services to be identified with the 'bad' prisoner, making it harder for those services to be provided.

The result of these splitting mechanisms - as seen in the context of service provision, training and research - is that the psychotherapeutic treatment of forensic patients will not be done by forensic psychiatrists, the professionals who generally run and direct the services. However, their attitudes to psychotherapy will determine how much will be available to those who need it. For example, few would dispute that the treatment of choice for sex offenders is mutative psychotherapy. (I do not exclude the anti-androgens from treatment, only suggest that they are not the first line of treatment.) A sex offender, or someone with similar pathology, would be referred appropriately to a forensic service, yet the bulk of treatment of such offenders is done by other non-psychiatric professionals. There is very little such treatment available, given the current levels of concern about such offenders. The same 
arguments apply to anti-social and borderline personality disorders. An alternative view might suggest that such patients are not appropriate forensic referrals, and that other services should deal with nonpsychotic personality disordered offenders-but which?

One must ask how this situation can be. It appears that psychotherapy and mainstream forensic psychiatry are divided; not only in terms of training, but also in relation to research and service provision. It is particularly noticeable in relation to those forensic psychiatrists who feel that one can practise psychotherapy without being in therapy oneself; thereby diminishing the work itself, and the importance of their own feelings. There are several possible reasons.

First, our ignorance about some very fundamental questions. Without reviewing the entire debate about research into psychotherapy, it is clear there are difficulties about applying traditional scientific methods. This is particularly so given that outcome measures in forensic psychiatry are difficult to define. Are there really patients who are not, and never will be, suitable for psychotherapy? What are their characteristics? How does one deal with violence between therapist and patient, and still maintain the therapeutic relationship? Do groups work better for these clients than individual therapy? The research is yet to be done.

Second, it is arguable that psychotherapy is essentially, and only, client-orientated-unlike mainstream forensic work, which often involves third-party interests, the law being the most obvious. Unlike the United States, there is currently no Tarasoff-type ruling in Britain, and this can lead to differences of opinion about client confidentiality between the professional groups in question.

Finally, it is no secret that forensic psychotherapy is particularly demanding. The therapist must be closely in touch with the client's internal world, but not be overwhelmed by it. It is hard to digest the outrageous pain and rage that is often present. Without proper training and supervision it is not surprising that forensic psychiatrists are reluctant to get involved.

In my view, the forensic psychotherapist is very much an evolving species. Despite some pessimistic signs, it appears there is a real interest in providing psychotherapy to a much deprived client group. Like all evolving species, the forensic psychotherapist will probably have to survive some changes in climate, and make adaptations. However, in the end it is hoped that the species will be fruitful and multiply. 